COMMENT

https://doi.org/10.1057/s41599-020-0396-5

\title{
Ethics of quantification: illumination, obfuscation and performative legitimation
}

Siddharth Sareen (10 ${ }^{1,2 \star}$, Andrea Saltelli (i) ${ }^{2}$ \& Kjetil Rommetveit ${ }^{2}$

\begin{abstract}
The increasing use of quantification in all spheres of society is paralleled by the rise of digitalisation. These intertwining developments not only revolutionise data treatment, but also its societal effects. On the one hand, they have wonderfully enabling societal effects. On the other hand, they give rise to complex ethical dilemmas that motivate this call for an ethics of quantification. The central claim of this Comment is that quantification necessarily has two faces: illumination and obfuscation. Aspects that can be socially legitimated are illuminated, while those that cannot be so legitimated are obfuscated. This obfuscation poses ethical problems, hence its effects require rigorous analysis. Three ontologies of quantification are delineated to enable such examination: (i) as the disembodied practice of data processing in the 'ether'-this foregrounds elements of big data and artificial intelligence; (ii) as the situated practice and effects of quantification within societal contexts-this attends to governing subjects through numbers; and (iii) as increasingly incorporated in physical reality-this focuses on governmentality of behaviours and behavioural change as mediated through everyday objects through an 'Internet of Things'. Drawing on scholarship from the emerging sociology of quantification, the ethics of quantification is defined as the iterative illumination of obfuscation in legitimation by quantification. This is key for ensuring contextually desirable illuminating functions of quantification in all three ontologies.
\end{abstract}

\footnotetext{
${ }^{1}$ Department of Geography, University of Bergen, Bergen, Norway. ${ }^{2}$ Centre for the Study of the Sciences and the Humanities, University of Bergen, Bergen, Norway. *email: Siddharth.Sareen@uib.no
} 


\section{Introduction: The case for an ethics of quantification}

- ntering the 2020s, we live in an era marked by the ubiquity of quantification. Variants of the adage "not all that counts can be counted, but what is counted counts" have proliferated. Metrics accompany many of us in a bewildering array of activities. These range from the formal (giving satisfaction ratings after a meeting or hotel stay, submitting credit ratings to take on a mortgage, checking domestic electricity consumption when paying monthly utility bills) to the informal and everyday (tracking one's daily run speed and distance, reviewing new beers drunk at a bar on a smartphone application to notch up experience points, monitoring one's sleep patterns using a smart watch). They can be distant (tapping a smiley button to rate the experience of going through airport security protocols) or intimate (registering biometrics such as one's fingerprints to go through immigration border controls). They can be voluntary (a citizen filling out a national survey to feed their household characteristics into a demographics database) or coercive (public surveillance linked with a facial recognition database to fine jaywalkers). They can be clearly signposted (traffic counters along bicycle routes) or stealthy (sensors like WiFi signals that collect information such as purchase histories from proximate smartphones for efficient realtime targeted advertising at passers-by in public spaces). They can result in the production of a tangible number (e.g., an indicator, a rating) or decision, where the number crunching is out of sight, e.g., in facial recognition (O'Neil, 2016). The data, data points and pieces of information are increasingly incorporated into largescale information infrastructures operating across policy and everyday domains, as well as across territorial and sovereign borders. Streams of metrics along these spectra are constantly collected, processed and used in ways that we increasingly take for granted.

This unprecedented generation of data to metricise innumerable aspects of everyday life is paralleled by unprecedented computing power and technological sophistication to process such vast quantities of data (Savage, 2013). Data is organised into an assortment of data points, information, statistics and metrics by a wide variety of actors who deploy them for such versatile purposes that their direct and indirect effects are far from clear. What is resoundingly clear, however, is that these effects have significant political and ethical implications. Quantification of the self, combined with digitalisation on steroids, has systemic effects; it reconfigures intimate matters like healthcare not only for individuals but as a vital socio-economic sector acted upon at the aggregate level (Lupton, 2016). Prior distinctions between facts and values, so central to modern western societies, collapse in streams of raw data feeding algorithms that learn and reason, and connect data from unexpected sites and sources. The intermeshing of big data and algorithms increasingly blurs distinctions between different forms and indeed purposes of quantification. They cut across the infinitely small (i.e., nano-molecules) and the infinitely big (Planet Earth), and apply to seemingly any process, from education to traffic management to biomedical research. Berman and Hirschman (2018) enquire: "What qualities are specific to rankings, or indicators, or models, or algorithms?"

There is appetite to scrutinise quantification for perceived misuse of existing methodologies. For instance, the convulsions of significance testing in statistics have received wide attention (Amrhein et al., 2019), whereas mathematical modelling is a field with severe uncharted problems (Saltelli, 2019). Algorithms pose the risk of non-transparent, oft-proprietary tools used in decision-making and for policy support. Much quantification carries the conundrum that, without representing context and purpose of production, numbers can obfuscate as much as illuminate. Yet measurements generate traction for issues, hence acting within society often requires an appreciation of and entanglement with data politics (Bigo et al., 2019). The time is thus ripe for an ethics of quantification (Saltelli, 2020).

\section{Ethical dilemmas in the societal legitimation of quantification}

As Dencik et al. (2019) point out, the way data and society act upon each other is changing the meaning of justice, specifically data justice for society. Quantification is performative; it serves a legitimating function (Porter, 1996) because it can in principle illuminate and make hitherto intangible things commensurable. Ever increasing sophistication in the metricisation of data has reconstituted fields like global health in deep entanglement with financial markets and data management systems, quantifying attributes of bodies and populations in ways that impact the distribution of certainty and risk in healthcare (Adams, 2016). This raises the question of what basis actors use to claim commensurability. As a case in point, the Stat-Activisme movement in France aims to 'fight against' and 'fight with' numbers; it uses 'statistical judo' to expose the vacuity of existing metrics, and to statistically identify exclusion and neglect (Bruno et al., 2014). This can be read as an attempt to open the 'black box' of quantification and challenge its predominant modality of legitimation in terms of simply 'more data' or 'more efficiency' or 'improved competitiveness'.

An even more radical critique is offered by the French jurist Alain Supiot (2007), for whom the neoliberal market ideology has embraced quantification, so as to implement a sort of cybernetic, homoeostatic, horizontal society. This replaces the previous Fordist and Taylorist mechanical models. In the new model, the labour force is constantly mobilised though management by objectives. Here, numbers replace laws to create a world of dystopian injustice and dysfunction, where the only solution for individuals is to revert to a system of allegiance to the strongest actors (Supiot, 2007). This happens even as many (or most) of today's societal problems are of a nature that cannot be quantified, such as the consequences of species extinction, the impacts of a major terrorist attack, or the possible consequences of artificial intelligence (AI) systems that operate critical infrastructures going out of control. Neoliberal regimes of innovation and governance are premised on entrepreneurs actively embracing the incalculability and subjective perceptions of risk and uncertainty, following the epistemic principle to 'run-it-and-see-what-happens' (Rommetveit and Wynne, 2017) or Facebook's celebratedor execrated-'move fast and break things'.

Governance regimes that rely on quantification invariably use it to legitimate decisions and activities, such as resource allocation, and highlight that which is socially legitimatable (we define legitimatable to mean 'that which can be legitimated'). Yet where there is scope for interpretation, there is room for strategic manoeuvre. Hence there is a risk that things that are not socially legitimatable may be hidden, for instance when citizens are not able to register with a biometric system, such as Aadhaar in India, thus becoming invisible to and being made invisible by the biometricising state (Dandurand, 2019; Wevers, 2018). When such sleight of hand-even if unwittingly executed by a benevolent actor-is not detected and challenged, the effect of an impulse to quantify can be to distort what it quantifies and why.

For instance, any national government asking all its citizens to embed a chip in their wrist to share their real-time location is likely to be met with resistance despite assurances of maintaining anonymity in its use of data; yet a majority of citizens in most Western democracies today carry a smartphone on their person and consent to sharing their location with a number of applications owned by multinational private corporations on an everyday basis, most commonly to facilitate their live use of local maps. 
This ability to track location is routinely combined with a great deal of other background information (often by third parties) and constitutes a remarkably potent resource for commercial (and potentially other) uses within surveillance capitalism. Users consent to this, not merely to being users, but effectively also the products on sale, when they install smartphone applications. But the users are rarely if ever presented with a comprehensive and comprehensible explanation of such uses, which is relegated to the fine-print of the terms and conditions that apply. We want to use mapping services, but forcibly we also end up consenting to surveillance. The socially legitimatable use is highlighted; not socially legitimatable uses are obfuscated.

Markham et al. (2018) warn that a grand narrative around data analytics that accords truth value to knowledge based on objectivity claims and sheer volume can obscure processual human decisions. These authors forge ways to work with the awareness that, as algorithmic governance becomes ubiquitous, an accountability crisis is unfolding in the way societal interventions are deployed, based as they are on incomplete assumptions about big data. Not only do actors who perform algorithmic governance selectively play up its socially legitimatable uses, over time this mode of governance acts on society to expand the ambit of what can be socially legitimated. In Europe and globally, this is to some extent becoming recognised, for instance in up-scaled data protection frameworks, ethical guidelines for AI, and 'ethically aligned designs' of autonomous systems (IEEE, 2018). Yet, the question remains to what extent and at what speed such governance frameworks can remedy the democratic and regulatory deficits.

Never as today have the media been so determinant in accelerating the transactions taking place between science, technology, society and law (Saltelli and Boulager, 2020). For these authors, who look at the present with the lenses of Niklas Luhmann's social system theory, we are witnessing one social system-the media-irritating other systems, such as science, policy and technology up to the point of incapacitating some of the internal (autopoietic) functions of the systems themselves. When social media networks first came into being, few could have imagined sharing as much of their personal information online as many people do now without much consideration. This iterative trend feeds a quantification machine that in turn changes what personal means, and even who a person is. At such a juncture of flux, Hesse et al. (2019) think through the continuing relevance of qualitative data. They advocate for methodological diversity, contextualised and inclusive research, nuanced discussion of ethical dilemmas that transcend legality, translocal and transdisciplinary conversations, and responsible research and data infrastructures.

Research must now respond to the need to examine at depth the issues flagged by the forays discussed above. We frame this collective task as showing that analysis of the ethics of quantification can draw out, in various ways, how quantification obfuscates to legitimate. Understanding how such obfuscation happens and is performed can equip us with the means to confront it in practice, and to support the illuminating function of quantification in context-specific ways.

Whilst the present juncture intensifies the effect of quantification, our claim holds true at all times. The introduction of statistics and data collection on demographics from the mid-18th century onwards (Hacking, 1990) gradually eclipsed other ways of knowing and came to structure practices like national budgets, enabling key instruments of state power. While such quantification enhanced abilities for centralised planning, by the same token it eroded power at local scales where local knowledge was not, and could not be, quantified in national databases, but informed decisions through its everyday embodiment in the bodies of local planners. To take another noted example: John
Snow quantified and spatially mapped London's cholera deaths to reveal that the epidemic was linked to water from specific wells, a novel methodology at the time whose success led to wide acceptance. Laudable in itself, this instance is not innocent; it could have been used as a basis to avoid investing in preventive measures to safeguard against contamination in less affluent areas, citing the methodology as a means to spatially limit an epidemic in case it occurred there. The Aadhaar example is a case in point of institutionalising non-innocent metrics. Despite clear evidence of limited data infrastructure to support reliable use of biometrics for benchmarking, and consequent risks of misdistribution in public schemes (Dandurand, 2019) or exclusion of marginalised groups like hard-working labourers with "Lost fingers, damaged fingertips, and rubbed-off skin contours" that make fingerprints unreadable (Rao, 2013, p. 74), the programme is backed by the state at the cost of vulnerable people (Drèze et al., 2017).

A listing of similar examples of non-innocent use of quantification would invoke a rich body of literature related to the misuse of metrics (Muller, 2018), algorithms (O’Neil, 2016) and statistical and mathematical modelling (Saltelli, 2020). Take the role of numbers produced in the financial centres of computation and held responsible for the onset of the most recent recession (Porter, 2012; Wilmott and Orrell, 2017; Ravetz, 2008). Bold intellectual manoeuvres such as those of Steven Pinker (2018), that deluge the reader with a profusion of numbers and graphs to argue that humankind has never had it so good, that inequality is non-existent or irrelevant, and that racism is receding, may also be classified as non-innocent (Lent, 2018; Riskin, 2019). In this specific example, Pinker adopts an aggressive stance against the purported enemies of Enlightenment-a broad category spanning from Nietzsche to Pope Francis via the Frankfurt School, the New York Times, and the discipline of Science Studies. In doing so, Pinker mobilises genuine and quantified instances of progress to advance the author's political stance and worldview via the selective use of measures. As discussed in Saltelli (2020), a heterogeneous community increasingly perceives and indicts quantification as instrumental to domination, including technologists (Lanier, 2006), jurists (Supiot, 2007) and economists (Zuboff, 2019).

Thus, any exercise of quantification demands an ethics to situate it within a given social context, not least because quantification acts in reflexive relation to the object and the environment that is quantified (Sareen et al., 2020; Saltelli, 2020).

\section{A definition and three ontologies for the ethics of quantification}

Whether as citizens or as users, our bodies and lives are governed as subjects, yet within the new data economy we also occur as mere data sources. The expanding creep of quantification subtracts decisional space from the governed subjects to governing ones, reducing the space for democratic objections, which would instead question the desirability of the new practices. This new brand of quantification banks on the social legitimacy of the models it deploys as proxies for reality. Complex as these are, these artefacts of quantification are simplified representations of reality and thus liable to be flawed. Their defensibility is a sleight of hand justified by pragmatic necessity. Practicing an ethics of quantification entails constant awareness of its intrinsic nature as a legitimation device that must be subjected to scrutiny and held to account by an active societal movement of resistance (Bruno et al., 2014; Saltelli, 2020). We can thus define an ethics of quantification as the iterative illumination of obfuscation in legitimation by quantification. Only then can quantification perform its illuminating function. 
We heuristically distinguish between and address three ontologies of quantification:

- as the disembodied practice of data processing in the 'ether'; - as the situated practice and effects of quantification within societal contexts; and

- as increasingly incorporated in physical reality itself (through the ubiquitous use of sensors, radio frequency identification and the like).

The first ontology foregrounds elements of big data and AI; the second ontology attends to governing subjects through numbers; and the third introduces governmentality of behaviours and behavioural change as mediated through everyday objects and things through an 'Internet of Things'.

Foregrounding big data, AI and the increasing automation of tasks-which combine so that computing can feed off of vast quantities of societally generated data using trial and error, commonly referred to as machine learning-focuses our attention on the ethical concerns implicated in the practice of quantification itself. Life in the 2020s is permeated by advertisements, news feeds, information access, personal search histories, social media cookies and cloud-based personal data repositories. These artefacts render big data and AI pervasive to a degree that impacts the qualitative implications of quantification. By tapping into, acting upon and becoming imbricated into everyday infrastructures, quantification becomes co-constitutive of everyday life. It becomes an adaptive structuring force that interplays with institutional structures and individual agency (Supiot, 2007). Addressing how AI impacts cognitive capacity and privacy, and what is lost and gained in the process, is thus inextricably linked with the ethics of quantification. The sets of big data that feed AI are generated in societal contexts that feature deeply entrenched inequities and injustices. There is thus a risk of reinforcing effects, reifying tendencies and rigidifying divisions through vicious cycles. Hence, we are particularly interested in scrutinising how AI differs from societal traits such as prioritising and privileging 'similar others', and whether it reproduces or exacerbates them. A thorough understanding of these mechanisms has implications for standards and regulations around expanding AI and big data use.

Attending to the situated practice and effects of quantification within societal contexts and infrastructures places the focus on sociological and socio-material aspects of the ethics of quantification-which calls for the combined efforts of technologists, sociologists, jurists, statisticians and data scientists. This requires probing how citizens interact with quantification and become quantified subjects who are governed through numbers in vital aspects of their lives in incredibly powerful ways. It also requires tracing the networks and practices of increasingly coordinated transnational infrastructures that underpin the new 'data economy'. The modalities of quantification are typically decided at higher levels of decision-making than the individual level that is being quantified through data extraction, posing the question of whose seeing is enabled and empowered, and who is merely 'being seen' without the required mechanisms for checks and balances on the watchers (Sareen and Rommetveit, 2019). Major decisions often take place in a socio-spatially centralised manner, with little room for representation of individual concerns and preferences by the citizens they affect. There are thus elements of uneven scalar effects (in terms of both hierarchical levels and spatial scales) to consider in relation to modalities such as what is quantified about citizens as subjects and about everyday phenomena like consumption, metrical performance, environmental interaction and death.

We discern a need to articulate the relationship between measurement and subjecthood in order to understand the ethical implications of how quantification acts on its subjects. This means recognising as inherently political the very act of choosing quantifiable proxy variables that render particular characteristics of messy reality commensurable and then serve to represent them. This movement is not new, and has precedents in the decadal fight of sociologists and ecologists against the numerification of everything, whereby both society and the environment can be seen as subject to neat systems of prediction and control (Pereira and Funtowicz, 2015; Stirling, 2019, 2008). Whether it is representative or not, quantification provides an evidence base to make claims about how things are. In doing so, it impacts both public understandings and societal commitments to particular configurations of resource allocation. Unpacking its evolving modalities has thus become critical to engagement with the embodied socio-material conditions of life in the $2020 \mathrm{~s}$.

Received: 9 December 2019; Accepted: 17 January 2020; Published online: 31 January 2020

\section{References}

Adams V (2016) Metrics: what counts in global health. Duke University Press Amrhein V, Greenland S, McShane B (2019) Scientists rise up against statistical significance. Nature 567(7748):305-307

Berman EP, Hirschman D (2018) The sociology of quantification: where are we now? Contemp Sociol 47(3):257-266

Bigo D, Isin E, Ruppert E (2019) Data politics: worlds, subjects, rights. Routledge, London

Bruno I, Didier E, Prévieux J (2014) Stat-Activisme. Comment Lutter Avec Des Nombres. Zones, La Découverte, Paris

Dandurand G (2019) When biopolitics turn digital: transparency, corruption, and erasures from the infrastructure of rationing in Delhi. Political Leg Anthropol Rev 42(2):268-282

Dencik L, Hintz A, Redden J, Treré E (2019) Exploring data justice: conceptions, applications and directions. Inf Commun Soc 22(7):873-881

Drèze J, Khalid N, Khera R, Somanchi A (2017) Aadhaar and food security in Jharkhand. Econ Political Wkly 52(50):51

Hacking I (1990) The taming of chance (Cambridge University Press)

Hesse A, Glenna L, Hinrichs C, Chiles R, Sachs C (2019) Qualitative research ethics in the big data era. Am Behav Sci 63(5):560-583

IEEE (2018) Ethically aligned design: a vision for prioritizing human well-being with autonomous and intelligent systems. The IEEE Global Initiative on Ethics of Autonomous and Intelligent Systems

Lanier J (2006) Who owns the future? Penguin Books

Lent J (2018) Steven Pinker's ideas are fatally flawed. These eight graphs show why. open Democracy

Lupton D (2016) The quantified self. John Wiley \& Sons

Markham AN, Tiidenberg K, Herman A (2018) Ethics as methods: doing ethics in the era of big data research-Introduction. Soc Media+ Soc 4 (3):2056305118784502

Muller J (2018) The tyranny of metrics. Princeton University Press

O'Neil C (2016) Weapons of math destruction: how big data increases inequality and threatens democracy. Random House Publishing Group

Pereira A, Funtowicz S (2015) Science, philosophy and sustainability: the end of the Cartesian dream. Routledge

Pinker S (2018) Enlightenment now: the case for reason, science, humanism, and progress. Random House

Porter T (1996) Trust in numbers: the pursuit of objectivity in science and public life. Princeton University Press

Porter T (2012) Funny numbers. Cult Unbound 4:585-598

Rao U (2013) Biometric marginality: UID and the shaping of homeless identities in the city. Econ Political Wkly 48(13):71-77

Ravetz J (2008) Faith and reason in the mathematics of the credit crunch. Oxford Magazine

Riskin J (2019) Pinker's Pollyannish philosophy and its perfidious politics. Review of Books, Los Angeles

Rommetveit K, Wynne B (2017) Technoscience, imagined publics and public imaginations. Public Underst Sci 26(2):133-147

Saltelli A (2019) A short comment on statistical versus mathematical modelling Nat Commun 10:3870

Saltelli A (2020) Ethics quantification or quantification ethics? Futures 116:102509

Saltelli A, Boulanger P (2020) Technoscience, policy and the new media. Nexus or vortex? Futures 115:102491

Sareen S, Rommetveit K (2019) Smart gridlock? Challenging hegemonic framings of mitigation solutions and scalability. Environ Res Lett 14(7):075004 
Sareen S, Thomson H, Tirado Herrero S, Gouveia JP, Lippert I, Lis A (2020) European energy poverty metrics: scales, prospects and limits. Glob Trans $2: 26-36$

Stirling A (2019) How politics closes down uncertainty-STEPS Centre. STEPS Centre

Stirling A (2008) "Opening up" and "closing down" power, participation, and pluralism in the social appraisal of technology. Sci Technol Hum Values 33 (2):262-294

Supiot A (2007) Governance by numbers: the making of a legal model of allegiance. Oxford University Press

Savage M (2013) The 'social life of methods': a critical introduction. Theory Cult Soc 30(4):3-21

Wevers R (2018) Unmasking biometrics' biases: facing gender, race, class and ability in biometric data collection. J Media Hist 21(2):89-105

Wilmott P, Orrell D (2017) The money formula. Wiley \& Sons

Zuboff S (2019) The age of surveillance capitalism: the fight for a human future at the new frontier of power. PublicAffairs

\section{Acknowledgements}

The authors are grateful for support from the Centre for the Study of the Sciences and the Humanities, University of Bergen, which made this contribution and collaboration possible.

\section{Competing interests}

The authors declare no competing interests.

\section{Additional information}

Correspondence and requests for materials should be addressed to S.S

Reprints and permission information is available at http://www.nature.com/reprints

Publisher's note Springer Nature remains neutral with regard to jurisdictional claims in published maps and institutional affiliations.

\section{(c) (1)}

Open Access This article is licensed under a Creative Commons Attribution 4.0 International License, which permits use, sharing, adaptation, distribution and reproduction in any medium or format, as long as you give appropriate credit to the original author(s) and the source, provide a link to the Creative Commons license, and indicate if changes were made. The images or other third party material in this article are included in the article's Creative Commons license, unless indicated otherwise in a credit line to the material. If material is not included in the article's Creative Commons license and your intended use is not permitted by statutory regulation or exceeds the permitted use, you will need to obtain permission directly from the copyright holder. To view a copy of this license, visit http://creativecommons.org/ licenses/by/4.0/.

(C) The Author(s) 2020 